\title{
Laboratory Generation of Multi-Sines with Pre-described Statistics
}

\author{
Nuno Borges Carvalho and José Carlos Pedro \\ Instituto de Telecomunicações, \\ Campo Universitário, 3810-193 Aveiro, Portugal \\ nborges@ieee.org
}

\begin{abstract}
This paper presents a laboratory instrument for the generation of RF multi-sines. The integration of an adaptive pre-distortion algorithm into a simple arbitrary waveform generator enabled the correct stimuli generation according to the previously developed theory.
\end{abstract}

\section{INTRODUCTION}

Tests of wireless systems for nonlinear behaviour, and modelling of wireless components, have been demanding for signals specially designed to fulfil certain statistical characteristics.

One of those signals is the widely used multi-sine excitation. It combines a sum of sine waves of different frequencies, but phase-correlated to each other. This correlation is a must from both the measurement and the data analysis viewpoints, since it enables the identification of each output and input component using the Discrete (or the Fast) Fourier Transform, DFT (FFT).

Although multi-sines have, for long, been used in active device characterization and modelling [1-6], their laboratory generation has yet not received the attention it deserves. For instance, there are several works [4-6] in which no restrictions are imposed to the phase of each tone in the multi-sine pattern. So, there can no control of even basic signal properties as the amplitude probability density function, $p d f$.

Recently, new theoretical algorithms to obtain multisine signals with desired statistics have been proposed $[1,2]$. These algorithms allow the RF design engineer to generate a multi-sine with some prescribed higher order statistics, at least in the mathematical description and/or in the simulator computer engine. That statistical behaviour is normally achieved for a pre-determined phase and amplitude arrangement of each tone.

Similar approaches have also been studied and proposed for multi-sines with minimum crest factors, a goal also achieved by controlling the phase and amplitude arrangements.
The authors of this paper have recently established the theoretical framework for the solution of a problem of great engineering significance by proving that the response's power spectral densities of an arbitrary (causal, stable and of finite memory) nonlinear dynamic system to two different signals are equal if and only if the two signals have equal higherorder autocorrelations or power spectral density functions.

Thus, they proposed special algorithms to design a standard signal stimulus - a multi-sine - capable of mimicking the test of a nonlinear dynamic system under a pre-determined excitation $[1,2]$. Then, they also proposed a numerical method for designing a multi-sine that shows such higher-order statistics, which established the methodology necessary to conceive an "ideal RF arbitrary waveform generator".

Nevertheless those results are only valid if the laboratory generation schemes allow the correct phase relation to be unaltered throughout the generator's RF path. If that is not the case, the actually obtained signal statistics and/or crest factor can differ significantly from the designed ones.

When these multi-sine signals are to be created by a real laboratory instrument, several problems appear which can be traced back to the nonlinear and dynamic path from the DSP generator to the output of the RF generator itself (see Fig.1).

As the multi-sine flows through the mixers, amplifiers, and filters, the tone amplitudes and relative phases are modified, necessarily impairing the required signal statistics.

If those changes result in mere linear phase shift and amplitude attenuation, then they can be compensated by including that effect in the DSP as a linear equalizer. However, if these effects are nonlinear, then the referred phase shift and attenuation depend on the amplitude of the multi-sine envelope, and a "nonlinear equalizer" (some form of dynamic linearizer) should be used. A microwave multisine waveform generator that incorporates such a dynamic linearizer is the object of this paper. 


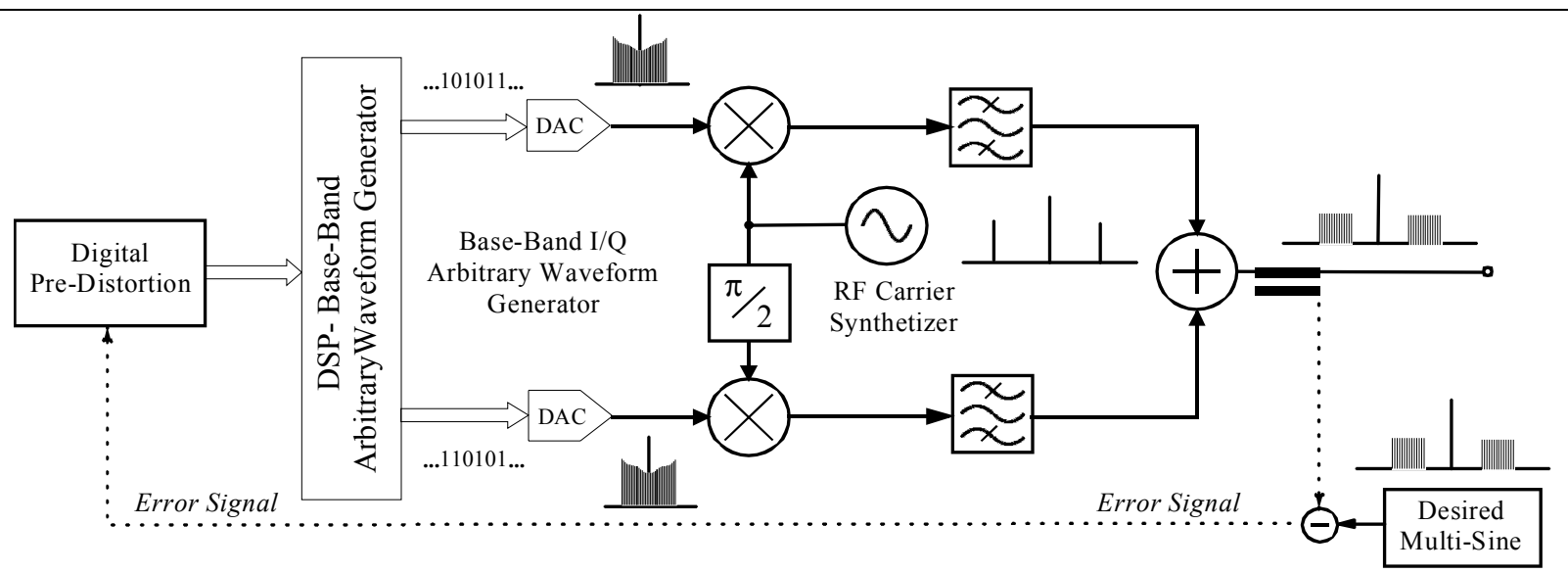

Fig. 1 - Block diagram of the proposed multi-sine generator.

In order to achieve that goal, in Section II, an algorithm based on envelope tracking is presented, while, in Section III, several simulated results are given. Finally, some conclusions will be drawn.

\section{ENVELOPE TRACKING BY HARMONIC BALANCE}

The idea subjacent to this paper is to design and implement a real arbitrary waveform generator capable of overcoming the limitations mentioned above. For that, we will compare the actual output multi-sine with the desired one, and generate an error signal that will be used to dynamically adjust the magnitudes and phases of the DSP synthesized multi-sine up to a point where the error is small enough. The optimization algorithm is based on the harmonic balance, HB, engine commonly used in the simulation of RF/Microwave nonlinear circuits [3].

In its original form, the HB starts from the a-priori knowledge of the frequency position of the signal components to then balance the amplitude and phase of the positions corresponding to each frequency at all circuit nodes. In the present case our idea is similar, except that now we perfectly know what we must get in terms of phase and amplitude for each tone. So, the algorithm will work on a conceptual port where the error signal between the actually generated and the desired multi-sine is built. Then, by using an optimization technique, the amplitudes and phases of the DSP synthesized multi-sine will be changed to obtain the sought multi-sine. Expression (1) presents the HB system of equations,

$$
\begin{aligned}
& f(A, \theta)= \\
& =\left[\sum_{q=1}^{Q} A_{D S P q} \cos \left(\omega_{q} t+\theta_{D S P q}\right)-\sum_{q=1}^{Q} A_{S Y N T q} \cos \left(\omega_{q} t+\theta_{S Y N T_{q}}\right)\right]=\varepsilon
\end{aligned}
$$

where $x_{D S P}(t)=\sum_{q=1}^{Q} A_{D S P q} \cos \left(\omega_{q} t+\theta_{D S P q}\right)$

and $x_{S Y N T}(t)=\sum_{q=1}^{Q} A_{S Y N T q} \cos \left(\omega_{q} t+\theta_{S Y N T q}\right)$

are the digital multi-sine downloaded to the DSP synthesizer and the multi-sine obtained at the analogue output of the generator itself, respectively, $A_{D S P q}$ and $\theta_{D S P q}$ is the amplitude and the phase of the multi-sine at the output of the DSP and $A_{S Y N T q}$ and $\theta_{\text {SYNTq }}$ is the amplitude and the phase of the multisine at the output of the generator.

The idea is to minimize the value of the error $\varepsilon$, in order to obtain the desired multi-sine.

At the output of our generator we must sense the signal in order to measure $x_{S N N T}$. That can be done by using a sampling oscilloscope, or a microwave transition analyzer (MTA).

Since in the multi-sine case the signals are synchronous to a known reference, we can also use the usual DFT or one of its fast algorithms, the FFT, to calculate the amplitudes and phases of the measured time domain signal and then input these values into a computer that will control the DSP. The iteration of this process will allow us to generate the sought multi-sine very efficiently, as seen in the block diagram of Fig. 1.

In fact, we can even minimize any spectral regrowth that is being generated at the RF-front-end itself, as the proposed control is actually implementing some form of adaptive feedback linearization.

\section{SIMULATED RESULTS}

Using the algorithm presented in [2], a 20 tone multi-sine, of a pre-described higher order statistics was designed. Fig. 2a) presents the probability density function, $p d f$, of the sought multi-sine, while Fig. 2b) shows the corresponding time domain waveform of that signal. 


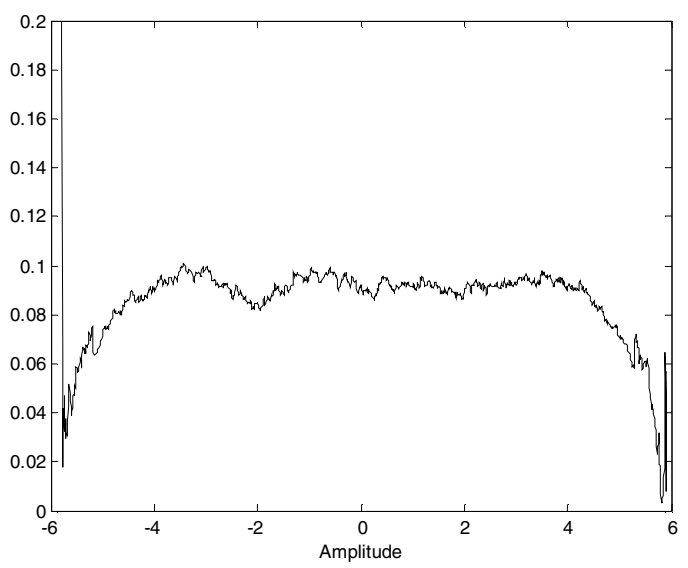

a)

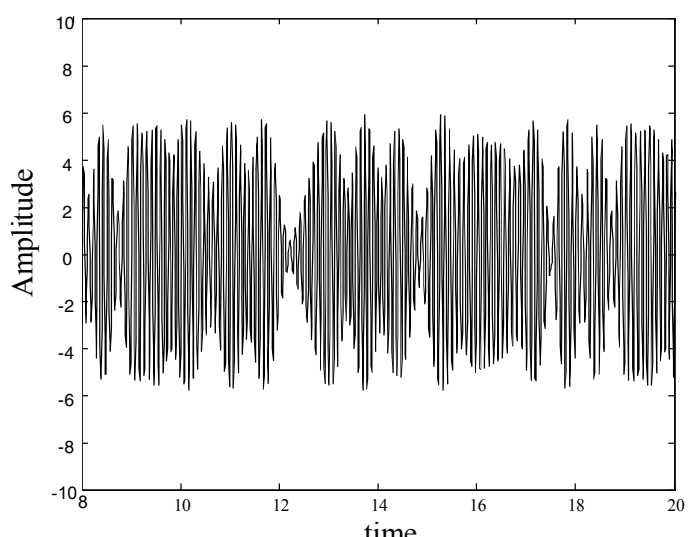

b)

Fig. 2 - Probability density function of the sought multi-sine (a) and time domain waveform (b).

After passing this multi-sine through a nonlinear generator RF front-end, the multi-sine $p d f$ and waveform became the ones represented in Fig. 3a) and b), respectively. Note the clear distortion of the $p d f$ caused by this non-ideal front end.

The proposed algorithm set-up was then applied to this system, and the final results are presented in Fig. 4a) and b), for the $p d f$, and time domain waveform of the synthesized multi-sine that will be fed to the DUT under test. Fig. 4c) and d) corresponds to the finally obtained multi-sine presented to the DSP generator, that despite is completely different from the one we sought by using the algorithm, it is the one that will present the synthesized multi-sine at the output of the generator.

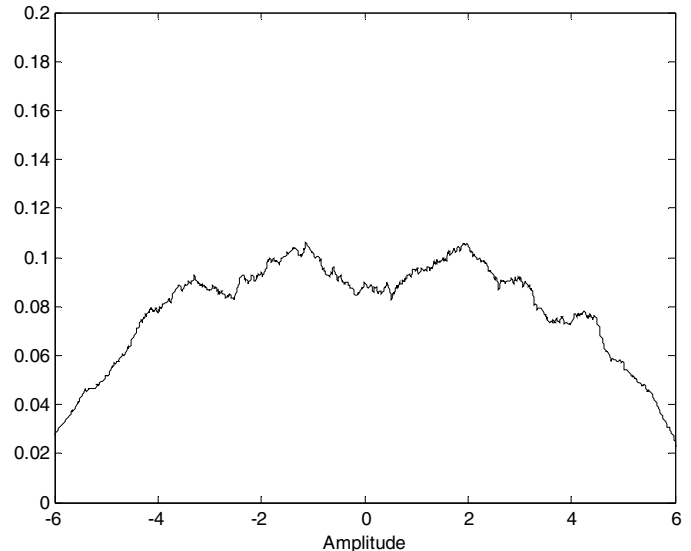

a)

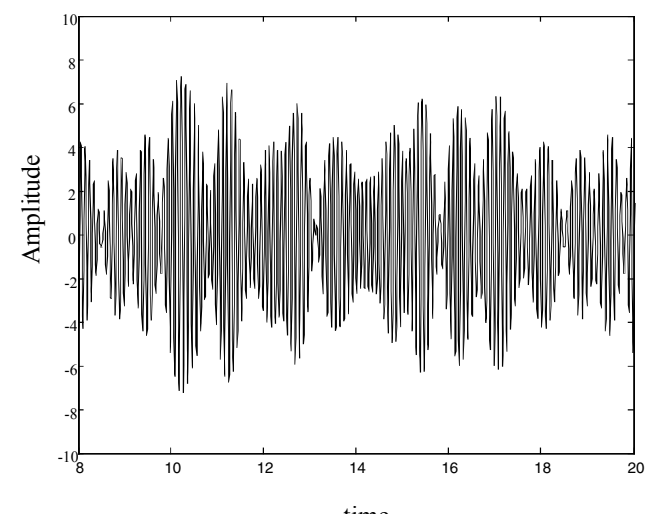

b)

Fig. 3 - Probability density function of the multi-sine after nonlinear distortion (a) and time domain waveform (b).

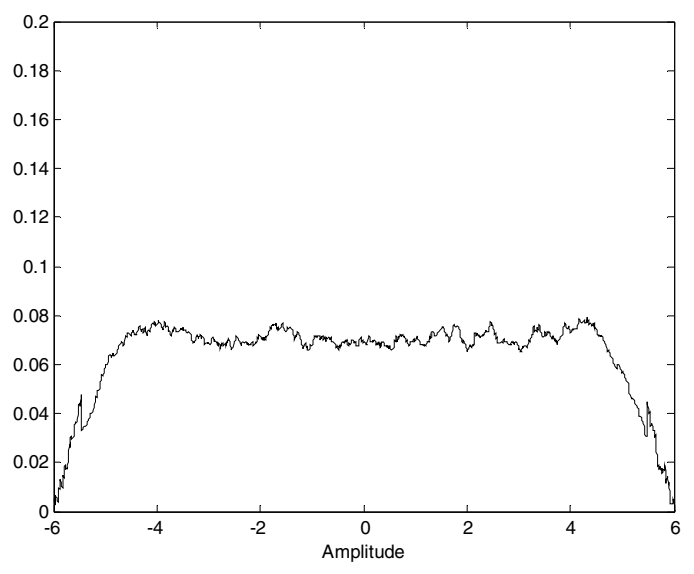

a) 


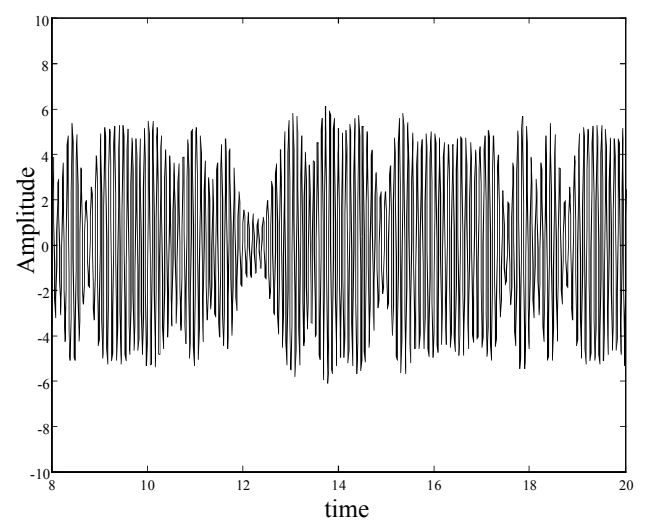

b)

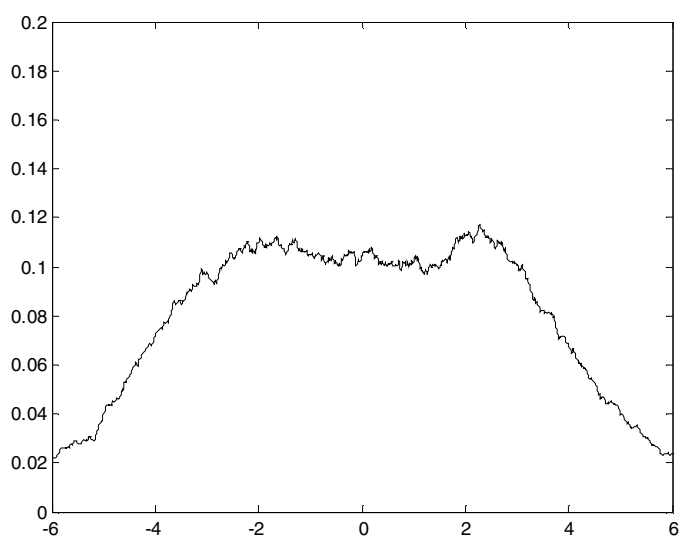

c)

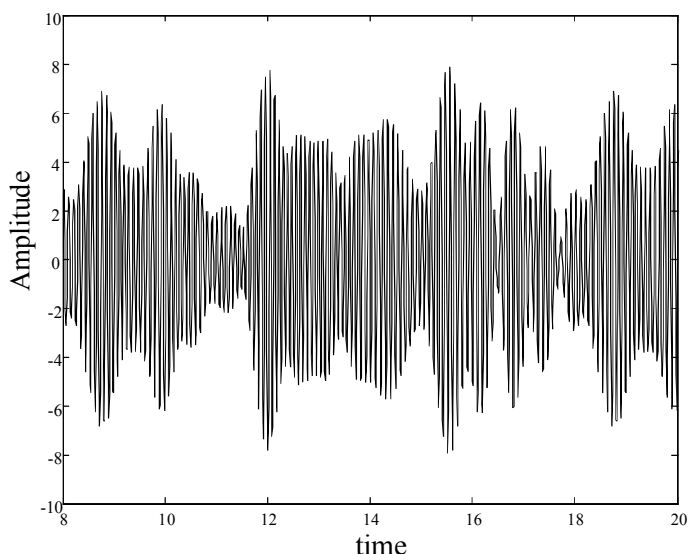

d)

Fig. 4 - Resulting multi-sine after the algorithm correction: (a) $p d f$ and (b) - output time domain waveform. Multi-sine signal synthesized by the DSP: (c) - $p d f$ and (d) - time domain waveform.
According to Fig. $4 \mathrm{c}$ ) and d), the proposed final multi-sine $p d f$ is completely different from the one initially desired. As seen from the output synthesized multi-sine, this is due to the pre-distortion correction necessary to compensate the dynamic nonlinearity of the generator.

The sampler of the time domain waveform can also be placed near the DUT, guaranteeing that the DUT sees exactly the higher order statistic proposed multi-sine.

\section{CONCLUSIONS}

In this paper a corrected waveform generator was proposed to generate a multi-sine with some prescribed higher order statistic and time domain waveform. This nonlinear dynamic pre-distortion overcomes the drawbacks associated to any form of linear or nonlinear distortions. This way, better multisine signals specially designed for RF characterization and/or modelling extraction are obtained.

\section{REFERENCES}

[1] J. C. Pedro and N. B. Carvalho, "Designing Band-Pass Multisine Excitations for Microwave Behavioral Model Identification", IEEE Intern. Microwave Symp. Dig., pp.791-794, Fort Worth, Jun. 2004.

[2] J. C. Pedro and N. B. Carvalho, "Designing Multisine Excitations for Nonlinear Model Testing", IEEE Trans. on Microwave Theory and Techniques, Vol. MTT-53, No 1 , pp.45-54, Jan. 2005.

[3] J. C. Pedro and N. B. Carvalho, Intermodulation Distortion in Microwave and Wireless Circuits, Artech House, Norwood, 2003.

[4] S. Boyd, "Multitone signals with low crest factor", Circuits and Systems, IEEE Trans. on Circuits and Systems, Vol. CAS-33, No. 10, pp.1018-1022, Oct. 1986.

[5] K. A. Remley, "Multisine excitation for ACPR measurements", IEEE Intern. Microwave Symp. Dig., ,pp.2141-2144, Philadelphia, Jun. 2003.

[6] A. R. Varkonyi-Koczy, "Synchronized multi-sine measurements via DSP methods", Instrumentation and Measurement Technology Conf IMTC-96. Proc. IEEE Vol. 2, 1996 pp.1056 - 1060. .. 